\title{
Evaluation of an online medicines' safety course for remote area nurses
}

AUTHORS

TOBIAS SPEARE BPharm MPH GDHR ${ }^{1}$

CHRIS RISSEL BSc(Psych) MPH PhD ${ }^{1}$

SUE LENTHALL BAdEd MPH\&TM PhD ${ }^{1}$

KATIE PENNINGTON BN GDRHP GCPHC ${ }^{1,2}$
1 Rural and Remote Health, Flinders University, Adelaide, South Australia, Australia

2 Edith Cowen University, Perth, Western Australia, Australia

\section{CORRESPONDING AUTHOR}

TOBIAS SPEARE Rural and Remote Health, Flinders University, PO Box 4066, Alice Springs NT 0871 Australia. Phone: +61 889514700 . Email: tobias.speare@flinders.edu.au

\section{ABSTRACT}

Background: Providing healthcare in a remote or rural setting can be complex and difficult, with many remote area nurses not receiving sufficient orientation or preparation. This is particularly important for the management of medicines.

Aim: This analysis evaluates an online medicines safety course called Pharmacotherapeutics for Remote Area Nurses.

Study Design and Methods: Eight cohorts of students ( $n=629$ ), between 2016-2020, were included in the evaluation. A mixed methods approach was used to evaluate the impact of the online pharmacotherapeutics course. At the end of each module and at the end of the course participants were asked to complete an online questionnaire. In-depth semi-structured interviews with key stakeholders $(n=9)$ were also conducted, and data thematically analysed.

Results: The online pharmacotherapeutics course was well received (average of 4.3 on a 5-point Likert scale). It was easy to navigate (2.6 on a 3-point Likert scale), the flow was logical (2.8 on a 3-point Likert scale) and it was clear how to achieve learning outcomes (2.8 on a 3 -point Likert scale). The learning content in each of the modules were well received (4.1-4.5 on a 5-point Likert scale) and deemed appropriate for nurses practicing in the remote areas (2.7-2.9 on a 3-point Likert scale). Thematic analysis of the stakeholder interviews and participant surveys revealed the pharmacotherapeutics course improved knowledge, confidence, and competence of nurses in relation to medicines management and results in better preparedness of the remote health workforce. Key stakeholder feedback highlighted that the pharmacotherapeutics course was seen as an important part of preparing and upskilling the remote health workforce.

Discussion: Overall, the pharmacotherapeutics course was well received by nurses and key informants. It improved self-reported knowledge, confidence, competence, and preparedness for nursing practice in remote locations.

Conclusion: The continued support of professional development and education for the remote health workforce is vital to ensure optimum patient care.

What is already known about this topic?

- People in remote Australia generally experience poorer health and face increased challenges in accessing care compared with major city counterparts.

- In remote regions, access to healthcare services is reliant on the availability of a competent workforce with nurses forming the backbone of rural and remote primary care.

- The use of medications is the most common intervention in healthcare and improvements in the quality use of medicines can have significant benefits to a person's wellbeing. 
What this paper adds:

- Professional development and education for the remote health workforce is vital to ensure optimum patient care.

- Education that is appropriate, relevant, and accessible can increase self-assessed knowledge, competence, and confidence in relation to medicines management.
- Online education that is developed in collaboration with stakeholders and addresses an identified need is an acceptable and accessible method of providing professional development to the remote health workforce.

\section{INTRODUCTION}

Australians living in remote areas generally experience poorer health than their major city counterparts. ${ }^{1}$ They have poorer access to health services and higher rates of avoidable hospital admissions. The poor health status of Aboriginal and Torres Strait Islander people is well recognised and accounts for a large portion of the increased burden of disease in remote areas. ${ }^{2}$ The 'gap' between Aboriginal and Torres Strait Islander and non-Indigenous people in Australia is not closing. ${ }^{3}$ Healthcare in remote Australia is a challenging and complex undertaking. ${ }^{4}$ Challenges related to geography, workforce maldistribution and instability, rural culture and cultural safety impair access to healthcare. ${ }^{5,6}$ There are far fewer doctors and allied health professionals in remote Australia compared to major cities.7 Nurses are the most evenly distributed health professionals across Australia and form the backbone of primary healthcare in remote Australia. ${ }^{8}$ Remote health services experience significant workforce instability characterised by extremely high annual turnover and poor retention..$^{9,10}$

Primary Health Care (PHC) clinics, located in remote communities and mainly staffed by Remote Area Nurses (RANs) and Aboriginal and Torres Strait Islander Health Practitioners and Workers, are the major providers of healthcare in very remote Australia. Many are part of Aboriginal Community Controlled Health Services governed by the communities that they serve. In most instances, PHC clinics are the first contact for all healthcare from emergencies to end of life care. The roles that RANs undertake are shaped by the remote context in which they work and live. ${ }^{11,12}$ Most work in very remote (MM7) Aboriginal and Torres Strait Islander communities, as designated by the Modified Monash Model (MMM) which measures remoteness from capital cities and population size on a scale from Modified Monash (MM) category MM 1 (major city) to MM 7 (very remote). ${ }^{13}$ Nurses in smaller facilities with less access and support from medical and allied health staff tend to have a broader scope of practice. As a health facility becomes more remote, RANs are more likely to take on a general practitioner (GP) substitute role, with support and collaboration from GPs, nurse practitioners and other experts via distance communication strategies or during periodic community visits. ${ }^{11}$ RANs are often the first point of care for patient presentations and as part of routine practice, employ a diverse set of skills encompassing the ability to examine, diagnose and provide treatments, including the supply of medications under clinical protocols. ${ }^{12}$ They diagnose and manage illness in line with clinical guidelines providing holistic care to people in their home communities. RANs manage medical presentations, including emergencies and trauma, stabilising patients before they are evacuated to definitive care supported by medical doctors on the end of a phone or video link. RANs provide age-appropriate 'wellness' checks to assist people in maintaining their health, including the detection, monitoring and assistance with selfmanagement of chronic disease. They undertake community development and health promotion activities following a PHC approach and conduct public health programs, including screening and surveillance, early intervention and prevention of illness advice. ${ }^{13}$

A significant difference in the scope of practice of RANs and nurses in non-remote settings is the management of medicines. Stowasser et al. describe the medicines management cycle, encompassing nine cognitive and physical steps from the decision to prescribe a medicine through to monitoring the impact of that medicine and three background processes (Figure 1). ${ }^{14}$ The medicines management cycle is a complex process that is fraught with risk. ${ }^{14}$ Across Australia, nurses consistently perform activities in relation to the management of medicines, mainly related to the administration of schedule 4 and schedule 8 medicines according to an order from an authorised prescriber. However, in the remote setting nurse roles with regards to medicines management include the initiation, supply, administration, and monitoring of medicines under clinical protocols. The regulatory mechanisms and required educational preparation for these roles varies considerably nationally. 15

The extended scope of practice of RANs, in addition to the challenges of the remote context and workforce instability, highlights the need for advanced knowledge and skills and accessible training. ${ }^{11,16,17}$ Effective orientation and continuing professional development is vital for ensuring an adequately skilled and professionally satisfied workforce. ${ }^{18}$ However, nurses new to the remote context often feel underprepared 


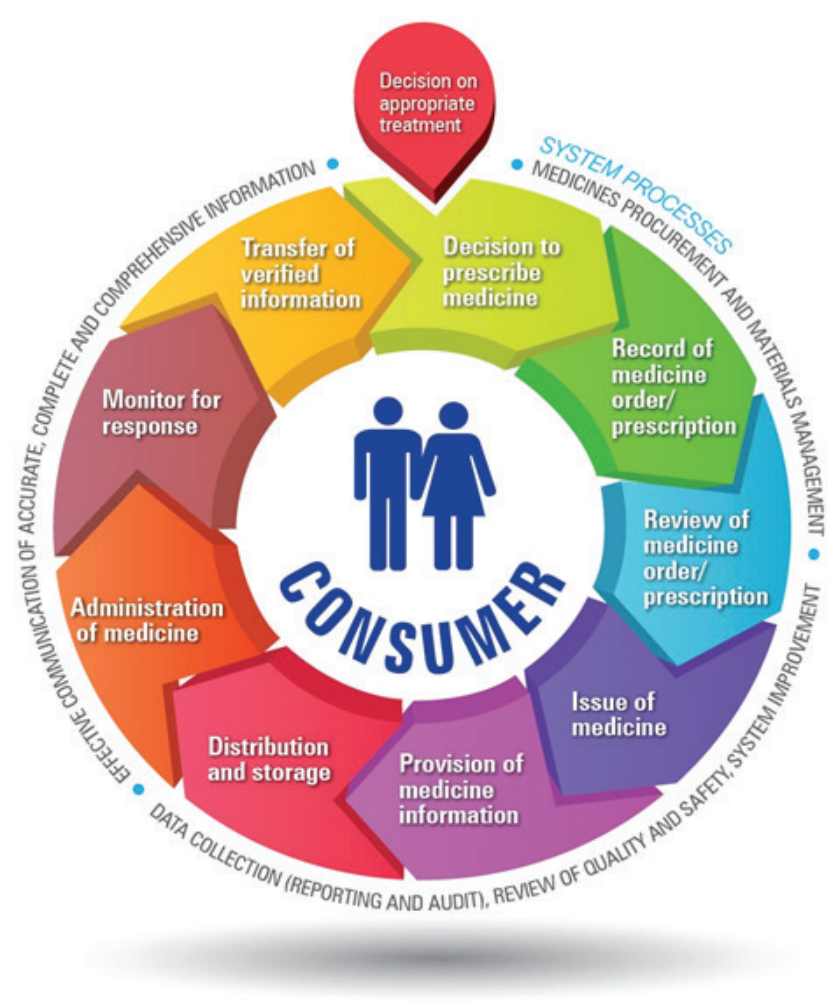

FIGURE 1: THE MEDICINES MANAGEMENT CYCLE

Ref: Adapted from Stowasser et al. ${ }^{14}$

by the training they receive and are overwhelmed by the demands of the RAN role. ${ }^{11,19-21}$ Many nurses felt the lack of orientation was a key issue related to responsibilities and expectations. Lenthall found that $30 \%$ of nurses in very remote areas had not received any orientation, and of those that did, less than half thought it was adequate. ${ }^{19} \mathrm{~A}$ recent survey by the professional organisation for remote health professionals found that only $51 \%$ of members had received an orientation that met their needs. ${ }^{22}$

\section{COURSE DEVELOPMENT}

'Pharmacotherapeutics for Remote Area Nurses' is the first online short course designed specifically for safe medicines management for the remote nursing workforce. It is designed to develop RANs' knowledge, skills, confidence, competence, and preparedness to ensure quality use of medicines within individual scope of practice. ${ }^{23}$ It was developed following extensive consultation and input from stakeholders, including state and territory Departments of Health, Aboriginal Community Controlled Health Services, workforce supply agencies, professional bodies, and RANs.

The pharmacotherapeutics course is based on adult learning principles and cognitive constructivist learning theory (Figure 2). ${ }^{24,25}$ The course comprises eight modules that are structured to build on participant knowledge and experience. The first two modules, RAN practice and pharmacology, provide a foundation for nursing practice in the remote context. The subsequent six clinical modules explore the management of conditions common to remote practice, including hypertension, type 2 diabetes, respiratory disease, mental health, infectious disease and antibiotic use, and pain management.

The pharmacotherapeutics course assists learners in developing the foundational skills necessary for clinical reasoning. Clinical reasoning is not just the acquisition of knowledge but includes collecting and analysing data and metacognition. ${ }^{26}$ While the acquisition of knowledge is vital for health professionals, the proper organisation of that knowledge is important for the development of clinical reasoning. New concepts are introduced gradually with activities scaffolded to provide an opportunity to apply concepts and principles to practical situations, including clinical scenarios and problem-based learning. ${ }^{27-29}$ The design of the pharmacotherapeutics course acknowledges that education of health professionals should structure information to facilitate the appropriate recall of key concepts and principles, using a framework to provide an organised approach to solving complex problems. Internationally and nationally endorsed frameworks, such as the World Health Organization Guide to Good Prescribing and the National Prescribing Service Prescribing Competencies Framework, are used to enable the critical assessment of evidence on individual merit in solving complex problems. ${ }^{30,31}$

Cognitive load associated with developing understanding of new concepts is reduced with full explanation of concepts, provision of extended readings and application of concepts in case studies that are reflective of real-life scenarios. ${ }^{25}$ Behavioural learning strategies, including the modelling of desired behaviours, are used to encourage learning outcomes. ${ }^{32}$

The pharmacotherapeutics course highlights the importance of culturally safe and responsive practice. Culture influences the way people view and experience health and illness and impacts on the beliefs, attitudes and decisions around access and engagement in healthcare. ${ }^{33}$ Culturally safe practice is critical in enhancing personal empowerment and, as a result, promotes more effective and meaningful pathways to self-determination for all providers and consumers of healthcare. ${ }^{34}$ Cultural safety and appropriateness of the course has been ensured through compliance with the Aboriginal and Torres Strait Islander Health Curriculum Framework. ${ }^{35}$ Through learning activities, such as discussion boards, self-reflective activities and the assessments, participants in the pharmacotherapeutics course are assisted in developing culturally safe and responsive practice. 


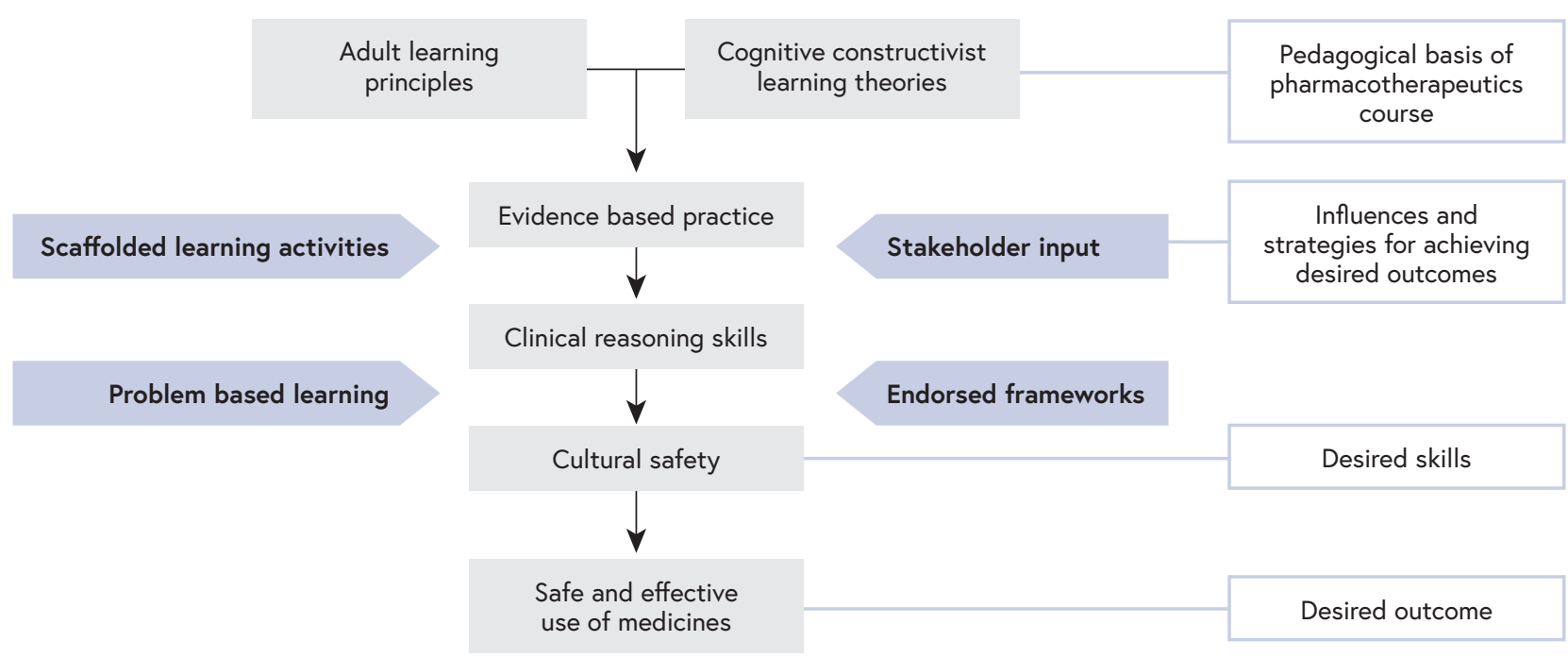

FIGURE 2: THEORETICAL MODEL, LEARNING STRATEGIES AND INFLUENCES IN DEVELOPMENT OF PHARMACOTHERAPEUTICS FOR REMOTE AREA NURSES ONLINE COURSE

\begin{abstract}
AIM
The aim of this paper is to describe process evaluation feedback of the pharmacotherapeutics course from participants, and to assess impact of the course on knowledge, confidence and competence and preparedness of nurses.
\end{abstract}

\section{METHODS}

A mixed methods approach was used to evaluate the impact of the online pharmacotherapeutics course. All nurses enrolled in the course from 2016 to 2020 were eligible to participate. At the end of each module, and at the end of the course participants were asked to complete an online questionnaire.

Following each of the eight modules participants were asked: 1) how they would rate the module, with responses corresponding to a 5-point Likert scale ranging from 1 (very poor) to 5 (excellent); 2 ) if the content of the learning module was appropriate for nurses in the remote context, responses corresponding to a 3-point Likert scale ranging from 1 (not appropriate) to 3 (appropriate); and 3 ) if they had any further comments.

At the end of the course participants were asked:1) how they would rate the course overall, with responses corresponding with a 5 -point Likert scale ranging from 1 (very poor) to 5 (excellent);2) were they able to access the course without problems, with responses corresponding with a 3-point Likert scale ranging from 1 (significant problems) to 3 (no problems);3) how they would assess the clarity of the navigation, expectations, and the interactive case study, with responses corresponding to a 3-point Likert scale ranging from 1 (not clear) to 3 (clear); and 4) how they would assess the logic of the flow, with responses corresponding to a 3-point Likert scale ranging from 1 (flow not logical) to 3 (flow logical).

Participants were also asked open ended questions on 1) what are the positive aspects of the pharmacotherapeutics course; 2 ) what are one or more things you will take away from completing this course; 3 ) how could the course be improved; and 4) are there other topics that you think should be included?.

An understanding of the impact of the pharmacotherapeutics course in relation to improving knowledge, confidence and competence and preparedness of nurses practicing in remote areas was assessed through semi-structured interviews with key stakeholders and content analysis of participant feedback. Key stakeholders with knowledge and insight into remote nursing workforce challenges, requirements and trends were purposefully recruited for interviews through professional networks. Stakeholders were asked to respond to "do you feel the online course is impacting on the knowledge, confidence, competence and preparedness of working in remote locations, and if so, how"?

Central Australian Human Research Ethics Committee granted ethical approval for the evaluation (HREC-16-441).

\section{ANALYSIS}

Data from the surveys were tabulated using Microsoft Excel. Descriptive analyses described the frequency of responses and average participant responses. Key stakeholder input via interview transcriptions and course participant questionnaire responses, was analysed thematically using Nvivo. ${ }^{36}$ The key themes from the analysis of interviews and participant responses were summarised. 


\section{RESULTS}

A total of 629 nurses from eight cohorts completed the end of course questionnaire, generating an overall response rate of $78 \%$. Respondents were employed by Government Departments of Health (46\%), employment agencies (28\%), Aboriginal Community Controlled Health Services (14\%), and other organisations (12\%).

The course content was rated well, with average approval rating per module of 4.1-4.5 on a 5-point Likert scale (Table 1 ). The content was deemed appropriate for nurses practicing in the remote areas, average rating per module of 2.7-2.9 on a 3-point Likert scale. The course was highly regarded, with overall average approval rating of 4.3 on a 5-point Likert scale. The majority of participants were able to access the course with minimal issues (2.6 on a 3-point Likert scale). They found the course was easy to navigate (2.6 on a 3-point Likert scale) and the flow of the course was logical (2.8 on a 3-point Likert scale). Respondents found that it was clear how to achieve learning outcomes (2.8 on a 3-point Likert scale). A significant proportion of respondents (57.8\%) experienced challenges with the case study, an automated clinical scenario that forms the final assessment of the pharmacotherapeutics course.

\section{QUALITATIVE RESULTS}

Participants found that the course improved practice, increased awareness, and resulted in better preparedness for practice in a remote area. Improved practice, particularly in relation to greater knowledge, enhanced confidence and improved competence resulted in better patient care. An increased awareness of scope of practice, resources to support practice, challenges of remote context and risks associated with medicines were also identified. Nurses undertaking the course prior to working in remote locations found it helpful in preparing them for the practice.
Participant quotes:

I found (the hypertension module) interesting and could relate this to my practice. The very next day I had a lady whose BP (blood pressure) was persistently high and was on an ACEi (angiotensin enzyme converting enzyme inhibitor), she had other co-morbidities. I spoke to the doctor and she popped her on a BB (beta blocker) for one week. Her BP stabilised and the reasoning and rationale along with the module confirmed what I had been learning, along with the doctor's reasoning.

Respect for complexity of clinical decisions. Accountability, therapeutic relationship, education, appreciation of the hazards, therapeutics of some groups of drugs.

More informed about the decision making process of how and why people are on the different medications for their chronic disease.

Participants also raised issues associated with the technical aspects of the online course, such as disruption of internet services in remote locations, weblinks not functioning and interactive aspects of the course, such as the case study, not functioning correctly. Some participants perceived the content as too advanced for a nursing role, reported a lack of access to recommended resources, and experienced challenges associated with timing of the courses.

Overall, the nine key stakeholder interviews highlighted that the pharmacotherapeutics course improved knowledge, confidence, and competence of nurses particularly in raising awareness of scope of practice and available resources.

Stakeholder interview quotes in relation to impacts on knowledge:

'I think that nurses have a greater level of knowledge, feel much more comfortable, and have confidence in giving medications from the CARPA (clinical procedure manual) after doing the course...'

\section{TABLE 1: PARTICIPANT RATINGS OF ASPECTS OF THE PHARMACOTHERAPEUTICS COURSE}

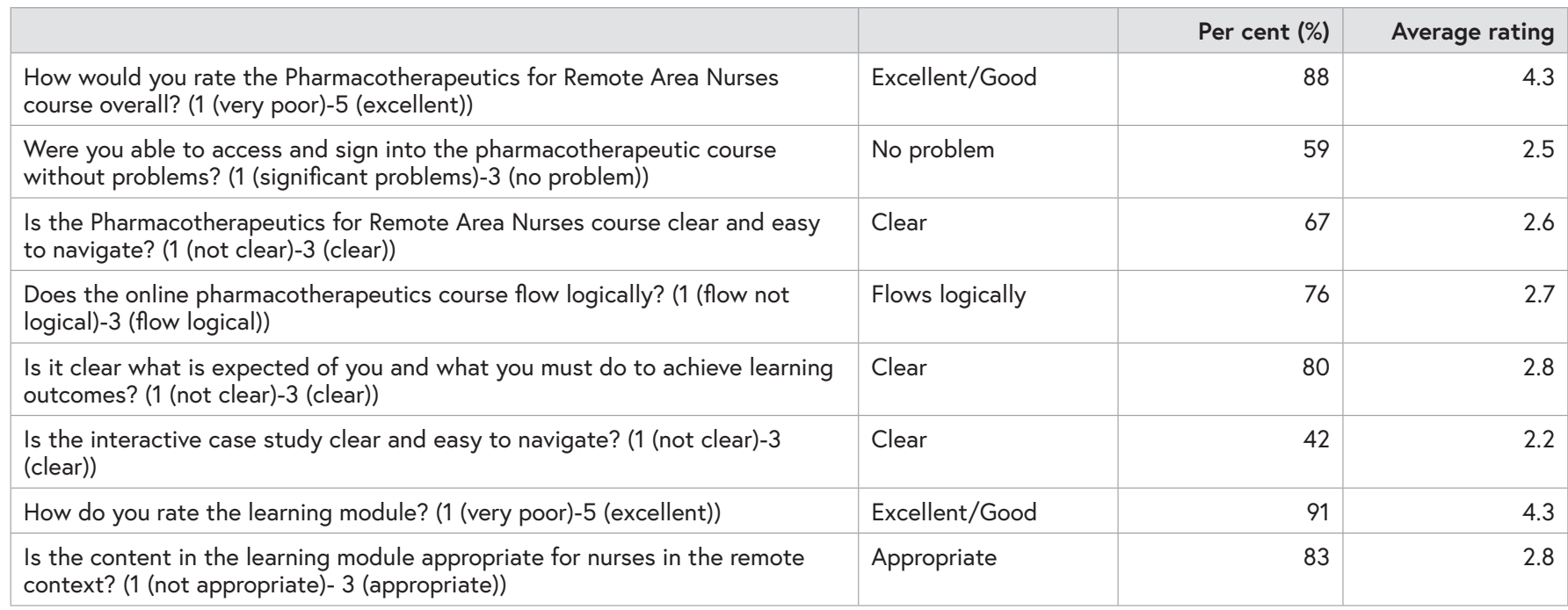




\section{Workforce Recruitment Agency.}

'(The pharmacotherapeutics course)... gives the nurses/ midwives a better understanding of medications, their use, their side effects, interactions with other medications,..' Director of Nursing and Midwifery

'(The pharmacotherapeutics course)... is a great refresher and provides essential knowledge learning base for the RAN working out of the CARPA manual (clinical procedure manual).' Remote Area Nurse Manager

Stakeholder interview quotes in relation to impacts on confidence:

'I do feel the course is impacting on nurses (confidence) as it transitions them to being an RN to a RAN, and instilling greater confidence to treat their patients with a firm knowledge base.' Workforce Recruitment Agency.

'(Nurses are).. more likely to ask me questions.' Aboriginal Community Controlled Health Service Pharmacist

'... knowledge builds confidence in all clinicians.' Remote Area Nurse Manager

Stakeholder interview quotes in relation to impacts on competence:

'The competency of understanding correct legislation and legal requirements. The knowledge pharmacological interaction, correct use of medication and the competency that goes with this.'

Workforce Recruitment Agency.

'(The pharmacotherapeutics course impacts competency)... and therefore the quality and hopefully continuity of care. Connected to increased knowledge confidence and experience.' Remote Area Nurses \& Peak Body

Stakeholder interview quotes in relation to impacts on preparedness to working in remote context:

'Legislatively it helps as it depends on the induction they receive in the clinic to whether they truly understand what they can and can't do. This course clarifies and allows a better understanding of their scope. It also gives them a heads up for maybe how much medication supply they are going to do. RN's who have not worked remote do not have an understanding of the volume and depth of medication knowledge potentially required in remote clinics.' Aboriginal Community Controlled Health Service Pharmacist

'There are many aspects of nursing that are unique to the remote setting. I believe the course does assist many nurses in their preparation to take on the challenges of medicines related practice in the remote setting.'

Department of Health Pharmacist

'In the remote context they (nurses) are working in advanced practice and courses like this are crucial.' Remote Area Nurse
Key stakeholder feedback informs us the

pharmacotherapeutics course is seen as an important part of preparing and upskilling the remote health workforce. Participants and key stakeholders identified a need for further opportunities to access professional development around medicines safety and pharmacotherapeutics.

\section{DISCUSSION}

Online courses can be effective in enhancing clinician knowledge and skills in relation to safe and effective use of medicines and reducing medication errors. ${ }^{17}$ Khalil \& Schliephake described the development of a medication safety course, which is similar to the development process of the Pharmacotherapeutics for Remote Area Nurses course. ${ }^{17}$ However, this publication does not include an evaluation of the impact of the online course on clinician knowledge. The evaluation of the pharmacotherapeutics course indicate that the online course improved self-reported knowledge, confidence, competence, and preparedness for nursing practice in remote locations. It was well received by nurses and key informants. This is important because the use of medications is the most common intervention in healthcare and improvements in the quality use of medicines can have significant benefits to a person's wellbeing. ${ }^{37}$ Problems with medicine use can occur at any time during the medicines management cycle, including when the decision is made to use a medicine, during dispensing, and while using the medicine. ${ }^{14}$ Medicines management usually involves multiple different health professionals adding additional layers of safety with each person involved in the process through repeated checks of the medication order, patient identity and patient alerts or medication interactions. ${ }^{8}$ In contrast, nurses in remote PHC centres often find themselves responsible for the entirety of the medication management cycle, which increases the risk of errors reaching the person and potentially causing harm. In situations where a single individual is responsible for all nine steps of the medication management cycle they need to be equipped with the necessary knowledge, skills, and competencies to ensure the safe and quality use of medicines. Clinician knowledge and skills are identified as contributing factors in incidence of medication errors. ${ }^{39}$ Education that equips nurses with knowledge and skills in safe medicines management, such as the pharmacotherapeutics course, are imperative in safeguarding and promoting the wellbeing of people who live in remote and rural areas of Australia. ${ }^{17}$

The Australian Nursing and Midwifery Accreditation Council accreditation standards were updated in 2019 to incorporate a standard referring specifically to the development of student knowledge and skills in pharmacotherapeutics and quality use of medicines (Standard 3.9), including the supply and administration of medicines. ${ }^{40}$ Prior to the change in accreditation standards educational programs did not necessarily have to include pharmacotherapeutics, which 
means that nurses who completed their undergraduate training prior to 2019 may not have received any formal education regarding the safe and appropriate supply of medications. The remote nursing workforce is slightly older than the national average, at 44 years vs 43 years, and is ageing faster, with $40 \%$ over 50 years compared to $33 \%$ nationally. ${ }^{41}$ This highlights the fact that few of the remote nursing workforce may have received formal training in relation to pharmacotherapeutics and the supply of medications, and hence the vital role that the Pharmacotherapeutics for Remote Area Nurses course can play in addressing this gap.

Pharmacotherapeutics for Remote Area Nurses has addressed a gap in professional development for the remote nursing workforce. While the pharmacotherapeutics course has been well received by participants and organisations involved in delivery of healthcare to remote locations in Australia, some deficiencies and challenges were identified. These included problems with technology and internet connectivity that were compounded by remote context and lack of IT support services. Docherty \& Sandhu's findings around student perceived barriers to online continuing professional development in primary care, including inadequate support, limited resources and course demands, reflected the experience of participants in the pharmacotherapeutics course. ${ }^{42}$ However the limited support described in the evaluation of the pharmacotherapeutics course tended to be related to IT and technological supports. Some participants in the pharmacotherapeutics course described the course material as too advanced or challenging, claiming "I am not a doctor". However, it is important to consider the advanced scope of practice of nurses in the remote context and the duty of care that health professionals owe to vulnerable people whom they are caring for and who often do not speak English as a first language and have low health literacy. RANs need to be confident and assured to question treatments that may be inappropriate, including medicine doses or combinations of medicines.

Participants and key stakeholders identified a need for further opportunities to advance knowledge and skills related to pharmacotherapeutics and safe use of medicine that is appropriate, relevant, and accessible to the remote nursing workforce. Future research could investigate the effect that amount of experience nursing in remote areas and experience with medicines management has on the impact of education on knowledge, confidence, competence, and preparedness to practice in remote areas.

\section{CONCLUSIONS}

The online medicines safety course, Pharmacotherapeutics for Remote Area Nurses, is well received and supported by nurses and key informants. It improves self-reported knowledge, confidence, competence, and preparedness for nursing practice in remote locations. The continued support of professional development and education for the remote health workforce is vital to ensure optimum patient care.

\section{REFERENCES}

1. Australian Institute of Health and Welfare. Rural \& remote health. Austalian Institute of Health and Welfare. 2019. [cited 2020 Sept 29]. Available from: https://www.aihw.gov.au/ reports/rural-health/rural-remote-health

2. Australian Institute of Health and Welfare. The health and welfare of Australia's Aboriginal and Torres Strait Islander peoples. Australian Institute of Health and Welfare. Canberra. 2015. [cited 2018 Apr 16]. Available from: https://www.aihw.gov.au/getmedia/584073f7-041e-48189419-39f5a060b1aa/18175.pdf.aspx?inline=true

3. Australian Indigenous HealthInfoNet. Overview of Aboriginal and Torres Strait Islander health status 2019. Australian Indigenous HealthInfoNet. Perth. 2020. [cited 2020 Jun 16]. Available from: https://healthinfonet.ecu.edu.au/keyresources/publications/39498/

4. Bourke L, Sheridan C. Understanding rural health - key concepts. In: Liaw ST, Kilpatrick K, editors. A textbook of Australian rural health. Canberra: Australian Rural Health Education Network; 2008

5. Brundisini F, Giacomini M, DeJean D, Vanstone M, Winsor S, Smith A. Chronic disease patients' experiences with accessing health care in rural and remote areas: a systematic review and qualitative meta-synthesis. Ont Health Technol Assess Ser. 2013;13(15):1-33.

6. Davy C, Harfield S, McArthur A, Munn Z, Brown A. Access to primary health care services for Indigenous peoples: a framework synthesis. Int J Equity Health. 2016;15(1):163

7. National Rural Health Alliance. Allied health workforce in rural, regional \& remote Australia factsheet. National Rural Health Alliance. Deakin West ACT. 2019. [cited 2020 Sept 29] Available from: https://www.ruralhealth.org.au/sites/default/files/ publications/fact-sheet-allied-health.pdf

8. Duckett S, Breadon P, Ginnivan L. Access all areas: new solutions for GP shortages in rural Australia. Grattan Institute. Melbourne. 2013. [cited 2016 Feb 16] Available from: https:// grattan.edu.au/report/access-all-areas-new-solutions-for-gpshortages-in-rural-australia/

9. Bourke L, Humphreys JS, Wakerman J, Taylor J. Understanding rural and remote health: a framework for analysis in Australia. Health Place. 2012;18(3):496-503.

10. Russell DJ, Zhao Y, Guthridge S, Ramjan M, Jones MP, Humphreys JS, et al. Patterns of resident health workforce turnover and retention in remote communities of the Northern Territory of Australia, 2013-2015. Hum Resour Health 2017;15(1):52.

11. Muirhead S, Birks M. Roles of rural and remote registered nurses in Australia: an integrative review. Aust J Adv Nurs. 2020;37(1):21-33. 
12. National Rural Health Alliance. Advanced nursing practice in rural and remote areas. National Rural Health Alliance. Deakin West. 2005. [cited 2020 Sept 18]. Available from: https://www. ruralhealth.org.au/sites/default/files/position-papers/positionpaper-05-07-01.pdf

13. Aitken R, Lenthall $S$, Mackay $B$. Rural and remote area nursing. In: Brown D, Edwards H, Buckley T, Aitken R, editors. Lewis's Medical-surgical nursing: assessment and management of clinical problems. 5th ed. Chatswood, NSW: Elsevier; 2020.

14. Stowasser DA, Allinson YM, O'Leary KM. Understanding the Medicines Management Pathway. J Pharm Pract Res. 2004;34(4):293-6.

15. Pennington K, Clark K, Knight S. A bitter pill to swallow; registered nurses and medicines regulation in remote areas of Australia. Rural Remote Health. 2020;20(4):6020.

16. Lenthall S, Wakerman J, Knight S. The frontline and the ivory tower: a case study of service and professional-driven curriculum. Aust J Rural Health. 2009;17(3):129-33.

17. Khalil H, Schliephake K. Design of an online medication safety module for clinicians. Int J Evid Based Healthc. 2017;15(2):63-8.

18. Humphreys J, Wakerman J, Wells R, Kuipers P, Jones J, Entwistle $P$, et al. Improving primary health care workforce retention in small rural and remote communities- how Important is ongoing education \& training? Australian Primary Health Care Research Institute. Canberra. 2007. [cited 2021 Aug 02] Available from: https://rsph.anu.edu.au/research/projects/ improving-primary-health-care-workforce-retention-small-ruraland-remote

19. Lenthall S. Back from the edge: reducing stress among remote area nurses in the Northern Territory [dissertation]. Adelaide: Flinders University; 2015.

20. Bennett P, Barlow $\vee$, Brown J, Jones D. What do graduate registered nurses want from jobs in rural/remote Australian communities? J Nurs Manag. 2012;20(4):485-90.

21. Mellor P, Greenhill J. A patient safety focused registered nurse transition to practice program. Contemp nurse. 2014;47(1-2):5160 .

22. CRANAplus. 2019 CRANAplus member survey. CRANAplus Magazine. 2020;117(4).

23. Speare T. Teaching remote area nurses. Australian Pharmacist. 2014;33(11):20.

24. Knowles MS. The modern practice of adult education: andragogy versus pedagogy. J Ext. 1971;9(1):60.

25. Biggs J, Tang C. Teaching for quality learning at university. 4th ed. Berkshire, England: Open University Press; 2011.

26. Cutrer WB, Sullivan WM, Fleming AE. Educational strategies for improving clinical reasoning. Curr Probl Pediatr Adolesc Health Care. 2013;43(9):248-57.

27. Salmon G. E-tivities: the key to active online learning. 2nd ed. New York, NY: Routledge; 2013.

28. Sweller J. Human cognitive architecture. In: Jonassen D, Spector M, Driscoll M, Merrill M, van Merrienboer H, editors. Handbook of research on educational communications and technology. 3rd ed. New York, NY: Taylor \& Francis Group; 2008.

29. Biggs J. Aligning teaching for constructing learning. High Educ Pedagog. 2003:1-4. [cited 2016 Apr 21] Available from: https:// www.heacademy.ac.uk/sites/default/files/resources/id477 aligning teaching for constructing learning.pdf
30. de Vries T, Henning R, Hogerzeil H, Fresle D. Guide to good prescribing: a practical manual. World Health Organization. Geneva. 1994. [cited 2016 Feb 17] Available from: http://apps. who.int/medicinedocs/pdf/whozip23e/whozip23e.pdf

31. National Prescribing Service. Competencies required to prescribe medicines: putting quality use of medicines into practice. National Prescribing Service. Sydney NSW. 2012. [cited 2016 Apr 21] Available from: https://www.heacademy.ac.uk/ sites/default/files/resources/id477 aligning teaching for constructing learning.pdf

32. Boghossian P. Behaviorism, constructivism, and socratic pedagogy. Educ Theory. 2006;38(6):713-22.

33. Curtis E, Jones R, Tipene-Leach D, Walker C, Loring B, Paine $\mathrm{SJ}$, et al. Why cultural safety rather than cultural competency is required to achieve health equity: a literature review and recommended definition. Int J Equity Health. 2019; 18, 174.

34. Indigeneous Allied Health Australia. Position paper: culturally responsive health care. Indigeneous Allied Health Australia. 2013. [cited 2016 Oct 27] Available from: https://iaha.com. au/wp-content/uploads/2020/02/Cultural-Safety-ThroughResponsive-Health-Practice-Position-Statement.pdf

35. Department of Health. Aboriginal and Torres Strait Islander health curriculum framework. Australian Government. Canberra. 2014. [cited 2020 May 19] Available from: https://www.health. gov.au/resources/publications/aboriginal-and-torres-straitislander-health-curriculum-framework

36. Braun V, Clarke V. Using thematic analysis in psychology. Qual Res Psychol. 2006;3(2):77-101

37. Roughead L, Semple S, Rosenfeld E. Literature review: medication safety in Australia. Australian Commission on Safety and Quality in Health Care. Sydney. 2013.

38. Pharmaceutical Society of Australia (PSA). Medicine safety: take care. Pharmaceutical Society of Australia. Canberra. 2019.

39. Brady A-M, Malone A-M, Fleming S. A literature review of the individual and systems factors that contribute to medication errors in nursing practice. J Nurs Manag. 2009;17(6):679-97.

40. Australian Nursing and Midwifery Accreditation Council. Registered nurse accreditation standards 2019. Australian Nursing and Midwifery Accreditation Council. Canberra. 2019.

41. Lenthall S, Wakerman J, Opie T, Dunn S, Macleod M, Dollard M, et al. Nursing workforce in very remote Australia, characteristics and key issues. Aust J Rural Health. 2011;19(1):32-7.

42. Docherty A, Sandhu H. Student-perceived barriers and facilitators to e-learning in continuing professional development in primary care. Educ Prim Care. 2006;17(4):34353. 